\title{
BMJ Open Scoping review of pharmacy-based initiatives for preventing unintended pregnancy: protocol
}

\author{
Philippa Buckingham (D), Natalie Amos (D), Safeera Yasmeen Hussainy, \\ Danielle Mazza
}

To cite: Buckingham $P$, Amos N, Hussainy SY, et al. Scoping review of pharmacy-based initiatives for preventing unintended pregnancy: protocol. BMJ Open 2020;10:e033002. doi:10.1136/ bmjopen-2019-033002

- Prepublication history for this paper is available online. To view these files, please visit the journal online (http://dx.doi. org/10.1136/bmjopen-2019033002).

Received 16 July 2019 Revised 24 November 2019 Accepted 03 January 2020
Check for updates

(C) Author(s) (or their employer(s)) 2020. Re-use permitted under CC BY-NC. No commercial re-use. See rights and permissions. Published by BMJ.

General Practice, Monash University Faculty of Medicine Nursing and Health Sciences, Notting Hill, Victoria, Australia

Correspondence to Professor Danielle Mazza; Danielle.Mazza@monash.edu

\section{ABSTRACT}

Introduction Due to a high global incidence of unintended pregnancy, finding novel ways to increase the accessibility of contraceptive products and information is critical. One proposed strategy is to use the accessibility of community pharmacies and expand the role of pharmacists to deliver these services. This protocol reports the methods of a proposed scoping review of pharmacy-based initiatives for preventing unintended pregnancy. We intend to identify the range of interventions employed by pharmacists worldwide and their outcomes and aim to infer the value of task sharing for reducing certain access and equity barriers to contraception.

Methods and analysis This protocol was developed with guidance from the Joanna Briggs Institute Methodology for Scoping Reviews. Reporting is compliant with the Preferred Reporting Items for Systematic Reviews and Meta-analysis (PRISMA) protocols. The scoping review will be reported according to the PRISMA Extension for Scoping Reviews. Seven electronic databases (PubMed, Ovid Medline, Embase, Cochrane Library, Scopus and Cumulative Index to Nursing and Allied Health Literature) were systematically searched for relevant literature published in English from 2000, on 22 August 2019. Two authors will individually screen articles for eligibility in Covidence and data will be charted and reported using a tool developed for the purpose of this review.

Ethics and dissemination Findings will be disseminated in publications and presentations with relevant stakeholders. Ethical approval is not required as we will be using data from publicly available literature sources. We will map available evidence across the breadth of studies that have been conducted and identify the effectiveness and acceptability of interventions.

\section{INTRODUCTION}

Access barriers to contraception contribute to continuing high levels of unplanned pregnancy internationally. ${ }^{2}$ The accessibility of both contraceptive products and information have equal weight as a high proportion of unintended pregnancies occur despite the use of a contraceptive method. ${ }^{34}$ Counselling may be important for selecting appropriate methods and continuation, ${ }^{5}$ while timely access is crucial for contraceptive regimes. The need for a medical prescription to obtain

\section{Strengths and limitations of this study}

- Scoping review methodology allows us to cover the broad topic area of pharmacy-based interventions for preventing unintended pregnancy to identify important areas for future research.

- A critical appraisal of the literature will be undertaken to highlight gaps in high-quality evidence.

- A limitation is that we will not be conducting an additional search of grey literature, which may exclude important sources of evidence.

contraception has been cited as a significant barrier. ${ }^{6}$ This may be due to the low availability of service providers, high combined costs of appointments and products and feeling burdensome consulting a clinician for minor health concerns such as contraception. $^{78}$

Peak bodies such as the WHO have recommended task sharing and increasing the use of pharmacists in sexual and reproductive health $(\mathrm{SRH})$ service provision. ${ }^{9}$ This strategy extends service accessibility to a subset of the population unwilling or unable to visit a clinician, with the potential to improve health service equity and reproductive health outcomes. ${ }^{10}$ Pharmacists present a number of opportunities for accessible contraceptive counselling, products, screening for sexually transmissible infections and medical abortion, and have demonstrated their capacity to deliver these services. ${ }^{11-13}$ Despite this, the findings in the literature pertaining to the feasibility, acceptability and effectiveness of unintended pregnancy prevention initiatives offered in a community pharmacy setting are extremely varied. ${ }^{8}{ }^{14}$ It is therefore difficult to determine what works, what services are appropriate and what strategies can be adopted to address these issues.

Researchers, policy-makers and healthcare professionals are challenged with equitable expansion of access to contraception 
services, irrespective of age, sexual orientation, gender identity, socioeconomic status or cultural or minority background. Internationally, the involvement of pharmacists in SRH is variable, as are the political, economic and social factors that shape health service delivery, overall population health and the effectiveness of these interventions. To date, an evidence map has not been created to report the range of outcomes of pharmacy-based strategies to prevent unintended pregnancies, despite a number of systematic reviews addressing interventions for specific populations and contraceptive products. ${ }^{815}$ A systematic review of pharmacy-based unintended pregnancy prevention initiatives was undertaken in the USA related to policy and practice, prior to the advent of pharmacist-prescribed contraception legislation in some states. ${ }^{14}$ Since this scope of practice expansion, a similar review has not been undertaken in the USA or internationally.

Scoping reviews are undertaken for a number of reasons, including to map evidence, determine the value of undertaking a systematic review, summarise and synthesise research findings for dissemination or identify research gaps. ${ }^{16}$ We report the methodology for a scoping review of pharmacy-based interventions for preventing unintended pregnancies. This is due to emphasis on publishing protocols for transparency and avoiding duplication. ${ }^{17}$

With this scoping review, we aim to report the range of outcomes of relevant services and interventionstheir feasibility, effectiveness and acceptability—and map evidence for pharmacists' scope of practice in unintended pregnancy prevention strategies. From this, we seek to determine the viability of task sharing for addressing access and equity issues to contraceptive information and products.

\section{METHODS AND ANALYSIS}

This protocol is reported in compliance with the Preferred Reporting Items for Systematic Reviews and MetaAnalysis (PRISMA) Protocols. ${ }^{18}$ The scoping review will be conducted with guidance from the the Joanna Briggs Institute (JBI) Methodology for Scoping Reviews ${ }^{17}$ and reported according to the PRISMA Extension for Scoping Reviews. ${ }^{20}$ The JBI Reviewer's Manual provides a contemporary methodological framework for the conduct of scoping reviews, for the purpose of facilitating knowledge translation in scientific and health research and pointof-care decision-making. This framework was chosen as the proposed scoping review is intended to inform health policy and evidence-based practice by community pharmacists. The steps to be employed as outlined in the JBI Reviewer's Manual are: (1) identifying the title and research question; (2) developing the inclusion criteria; (3) defining the search strategy; (4) study selection; (5) data extraction; (6) presentation of the results and (7) ethics and dissemination.

\section{Objectives}

The objective of the proposed review is to scope pharmacybased initiatives for the feasibility, acceptability and effectiveness for preventing unintended pregnancy.

This research is conducted within the National Health and Medical Research Council (NHMRC) Centre of Research Excellence in SRH for Women in Primary Care (SPHERE). The development of the SPHERE proposal involved a multidisciplinary team of national and international experts in SRH, primary care and pharmacy. SH is also a leading Australian pharmacy academic, having contributed to legislative reform for the emergency contraceptive pill. Collaborative input has informed the development of the research questions. These are based on the Population, Concept, Context (PCC) strategy.

The interventions we aim to assess are those relating to unintended pregnancy prevention (concept) for women (population) in the context of community pharmacy. This clarifies the scope of the literature that will be reviewed. ${ }^{17}$

The scoping review will map evidence pertaining to the following research questions:

1. What interventions for preventing unintended pregnancy have been adopted in a pharmacy setting?

2. What health and economic outcomes result from pharmacy-based unintended pregnancy interventions?

3. Are pharmacy-based unintended pregnancy prevention services feasible, accessible and appropriate?

\section{Inclusion criteria}

Development of the inclusion criteria is also guided by the PCC strategy. Articles eligible for inclusion are those published in English, as we do not have access to translation resources. The timeframe for inclusion will be articles published since 2000 as it was decided that the likelihood of finding meaningful data prior to this is low. This is justified by the only recent expansion of pharmacy into reproductive health management, with the recent availability of emergency contraception without prescription.

Pharmacy-based interventions are those defined as being undertaken by pharmacists, pharmacy assistants or other health professionals within a community pharmacy setting. Pharmacy-based interventions are eligible provided they involve a face-to-face interaction between patient and provider.

Interventions for women to prevent unintended pregnancy may be assessed objectively and from a range of stakeholder perspectives. Eligible participants are women of reproductive age (15-50) or pharmacists. Literature will be eligible if evaluating or describing pharmacy-based interventions for women with an unintended pregnancy prevention focus, the role of pharmacists in these interventions or feasibility issues for relevant pharmacy-based services. Other eligible articles are those describing the broader context of pharmacy-based unintended pregnancy prevention initiatives for women. Articles will be excluded if they assess sexual health services or products in a pharmacy setting without a focus on unintended pregnancy (eg, those addressing sexually transmissible 
Table 1 Search strategy (Ovid Medline: 1583 results)

\begin{tabular}{ll}
\hline Population: women & Female \\
\hline $\begin{array}{l}\text { Concept: unintended } \\
\text { pregnancy, birth control }\end{array}$ & $\begin{array}{l}\text { (pregnan* ADJ3 unintended or unplanned or unwanted or mistimed).mp OR (pregnan* ADJ3 (counsel* or } \\
\text { consult* or advice or information or educat*)).mp OR contracepti*.mp OR exp Contraceptive Agents/ OR } \\
\text { birth control.mp OR exp Contraception Behavior/ OR (contracepti* ADJ3 counsel* or consult* or advice or } \\
\text { information).mp }\end{array}$ \\
& $\begin{array}{l}\text { (Pharmacy.mp OR pharmaci* OR over-the-counter).mp OR exp Pharmacy Services/ OR OR Non- } \\
\text { Prescription Drugs/ OR Community Pharmacy Services/ OR Legislation, Pharmacy/ OR exp Health } \\
\text { Services Accessibility AND limit to yr="2000 -Current" AND limit to=English }\end{array}$ \\
\hline
\end{tabular}

infections) or reproductive health interventions not focused on pregnancy prevention (eg, medical abortion).

Outcomes of interest are feasibility (health system and provider barriers and facilitators), acceptability (assessed through provider and patient attitudes to pharmacy service provision), effectiveness (impacts on contraceptive uptake, behaviours and unintended pregnancy) and economic outcomes (cost-benefit, cost-effectiveness, quality of life indicators). Review outcomes will be refined iteratively during the review process.

To refine the scope of this review, the following literature sources are eligible for inclusion:

1. Intervention studies such as randomised controlled trials, cluster randomised trials, quasirandomised trials, pragmatic trials and pretest/post-test analyses.

2. Longitudinal studies measuring cohort or populationbased projections or impacts of relevant interventions.

3. Qualitative, quantitative and mixed-methods studies to measure and describe different aspects of pharmacybased unintended pregnancy services or interventions.

\section{Search strategy}

A preliminary search was undertaken in July 2019 (table 1). The search strategy was developed considering the research questions and PCC criteria above and refined with assistance from a medical librarian. It included various combinations and Medical Subject Heading (MeSH) terms for the following concepts: pharmacy, unintended pregnancy, contraception, contraceptive behaviours, contraceptive consultation and information. This was further refined as the authors become familiar with the literature and resources available. This methodology is favoured for scoping reviews as it improves sensitivity of the search. ${ }^{17}$ The final search was undertaken in August 2019.

\section{Study selection}

The search will comprise two levels: (1) title and abstract review and (2) full text review. Two authors, PB and NA, will independently assess articles for inclusion. A third reviewer will be involved to discuss and resolve any conflicts where a consensus cannot be reached between the first two authors. Seven electronic databases will be searched for eligible literature: PubMed, Ovid Medline, Embase, Cochrane Library, Scopus and Cumulative Index to Nursing and Allied Health Literature. Keywords from title and abstract review will be noted, and a second search will be conducted using these identified index terms and keywords. Forward searching will involve scanning reference lists of identified articles for additional papers of relevance, which will be screened for eligibility pertaining to the inclusion criteria.

\section{Data extraction}

A data collection tool will be developed to extract study characteristics. The literature will be charted by (but not restricted to): authors, year of publication, country, aims/ purpose, study population, sample size, methodology/ methods, intervention type, comparator details, outcomes and details of these and key findings that relate to one or more research questions. The charting table will be updated during the review should it be made apparent that additional information is relevant to the review objectives.

\section{Presentation of the results}

A table of the phases of the scoping review will be provided to detail the process of defining the research question, identifying and selecting articles, charting the data, summarising and disseminating our findings.

Due to heterogeneity in studies, methods and types of literature drawn on, a narrative approach will be taken when summarising and presenting the results. We will map available evidence across the breadth of studies that have been conducted and identify where these interventions were feasible, effective and acceptable to users and providers.

The main characteristics of intervention studies will be displayed in an evidence map, summarising findings that relate to the review outcomes by the type of service or intervention studied (over-the-counter contraception, emergency contraception, contraceptive counselling and so on). Evidence maps systematically collate and diagrammatically present available evidence on a broad topic area in a way that is accessible and usable. ${ }^{21}$ These are particularly relevant for identifying where evidence and gaps exist. ${ }^{22}$

For other types of literature, the main findings relating to one or more outcomes of interest will be summarised.

\section{Critical appraisal}

The JBI have developed a series of critical appraisal tools targeted towards specific study designs, including research syntheses, randomised and non-randomised trials, prevalence studies and economic evaluations. ${ }^{23}$ 
The critical appraisal tools each consist of a checklist and encourage nuanced reporting and interpretation of the critical appraisal findings instead of ascribing a grade for evidence quality. Critical appraisal of the literature will be undertaken at the time of data extraction. This step is important for the interpretation of the literature and identification of gaps in high-quality evidence. The findings of the critical appraisal will be presented with the findings from the literature to highlight new and developing areas in pharmacy practice that require future research and high-quality evidence to guide policy development and pharmacy practice.

\section{ETHICS AND DISSEMINATION}

Due to their considerable health, social and economic consequences, increasing access to services, products and information to prevent unintended pregnancies is of utmost importance. In the proposed scoping review, we will collate available evidence regarding the impacts of pharmacy-based reproductive health interventions and pharmacists' scope of practice in reproductive health. This will involve a secondary analysis of data collected in prior research, of which findings are publicly available. Ethical approval is therefore not required.

The scoping review will be undertaken as part of a larger project, designed to infirm decisions regarding the practice of community pharmacists in Australia. The findings of this scoping review will frame future research and facilitate knowledge translation activities for the role of pharmacists in women's reproductive health.

Findings will also be relevant to a number of stakeholders including researchers, pharmacists and other healthcare providers and the governing bodies responsible for health policy design and implementation. We are accountable to ensure the findings of this review reach all interested audiences. We plan to disseminate this research in publications and presentations and via SPHERE's National Communication Network.

\section{Twitter Safeera Yasmeen Hussainy @SafeeraHussainy}

Contributors PB designed the study and developed the methods supervised by NA, SYH and DM. DM guided the development of the review objectives, population context and concept and search strategy. NA was involved in developing the inclusion criteria and methods for study selection, data extraction and synthesis of the results. SYH contributed knowledge of pharmacy practice. PB, NA and DM met regularly to refine the protocol which was drafted by PB. All authors read, edited and approved the final manuscript prior to submission.

Funding This work was supported by the National Health and Medical Research Council (grant ID 1153592).

Competing interests DM has received research funding, travel grants and honorarium from Bayer.

Patient consent for publication Not required.

Provenance and peer review Not commissioned; externally peer reviewed.

Open access This is an open access article distributed in accordance with the Creative Commons Attribution Non Commercial (CC BY-NC 4.0) license, which permits others to distribute, remix, adapt, build upon this work non-commercially, and license their derivative works on different terms, provided the original work is properly cited, appropriate credit is given, any changes made indicated, and the use is non-commercial. See: http://creativecommons.org/licenses/by-nc/4.0/.

\section{ORCID iDs}

Philippa Buckingham http://orcid.org/0000-0001-9166-9719

Natalie Amos http://orcid.org/0000-0001-6558-2580

\section{REFERENCES}

1 Starrs AM, Ezeh AC, Barker G, et al. Accelerate progress-sexual and reproductive health and rights for all: report of the GuttmacherLancet Commission. The Lancet 2018;391:2642-92.

2 Bearak J, Popinchalk A, Alkema L, et al. Global, regional, and subregional trends in unintended pregnancy and its outcomes from 1990 to 2014: estimates from a Bayesian hierarchical model. Lancet Glob Health 2018;6:e380-9.

3 Richters J, Fitzadam S, Yeung A, et al. Contraceptive practices among women: the second Australian study of health and relationships. Contraception 2016;94:548-55.

4 Coombe J, Harris ML, Wigginton B, et al. Contraceptive use at the time of unintended pregnancy: findings from the contraceptive use, pregnancy intention and decisions study. Aust Fam Physician 2016;45:842-8.

5 Schivone GB, Glish LL. Contraceptive counseling for continuation and satisfaction. Curr Opin Obstet Gynecol 2017;29:443-8.

6 Dennis A, Grossman D. Barriers to contraception and interest in over-the-counter access among low-income women: a qualitative study. Perspect Sex Reprod Health 2012;44:84-91.

7 Michie L, Cameron ST, Glasier A, et al. Provision of contraception after emergency contraception from the pharmacy: evaluating the acceptability of pharmacy for providing sexual and reproductive health services. Public Health 2016;135:97-103.

8 Gonsalves L, Hindin MJ. Pharmacy provision of sexual and reproductive health commodities to young people: a systematic literature review and synthesis of the evidence. Contraception 2017;95:339-63.

9 The World Health Organization. Task sharing to improve access to family Planning/Contraception. Geneva, Switzerland, 2017. https:// www.who.int/reproductivehealth/publications/task-sharing-accessfp-contraception/en/

10 Rodriguez MI, Hersh A, Anderson LB, et al. Association of pharmacist prescription of hormonal contraception with unintended pregnancies and Medicaid costs. Obstet Gynecol 2019;133:1238-46.

11 Griffiths C, Miles K, Aldam D, et al. A nurse- and pharmacist-led treatment advice clinic for patients attending an HIV outpatient clinic. $J$ Adv Nurs 2007;58:320-6.

12 Gudka S, Marshall L, Creagh A, et al. To develop and measure the effectiveness and acceptability of a pharmacy-based Chlamydia screening intervention in Australia. BMJ Open 2013;3:e003338-e.

13 Puri MC, Harper CC, Maharjan D, et al. Pharmacy access to medical abortion from trained providers and post-abortion contraception in Nepal. Int J Gynecol Obstet 2018;143:211-6.

14 Farris KB, Ashwood D, Mclntosh J, et al. Preventing unintended pregnancy: pharmacists' roles in practice and policy via partnerships. J Am Pharm Assoc 2010;50:604-12.

15 Rafie S, Stone RH, Wilkinson TA, et al. Role of the community pharmacist in emergency contraception counseling and delivery in the United States: current trends and future prospects. Integr Pharm Res Pract 2017;6:99-108.

16 Arksey H, O'Malley L. Scoping studies: towards a methodological framework. Int J Soc Res Methodol 2005;8:19-32.

17 Aromataris E, Eds MZ. Joanna Briggs Institute reviewer's manual. The Joanna Briggs Institute, 2017. https://reviewersmanual. joannabriggs.org/

18 Moher D, Shamseer L, Clarke M, et al. Preferred reporting items for systematic review and meta-analysis protocols (PRISMA-P) 2015 statement. Syst Rev 2015;4:1.

19 Levac D, Colquhoun H, O'Brien KK. Scoping studies: advancing the methodology. Implementation Sci 2010;5:69.

20 Tricco AC, Lillie E, Zarin W, et al. PRISMA extension for scoping reviews (PRISMA-ScR): checklist and explanation. Ann Intern Med 2018;169:467-73.

21 Saran A, White H. Evidence and gap maps: a comparison of different approaches. Available: https://campbellcollaboration.org/media/k2/ attachments/6_Comparison_of_approaches_to_evidence_and_gap_ maps.pdf [Accessed 15 May 2019].

22 Schmucker C, Motschall E, Antes G, et al. [Methods of evidence mapping. A systematic review]. Bundesgesundheitsblatt Gesundheitsforschung Gesundheitsschutz 2013;56:1390-7.

23 The Joanna Briggs Institute. Critical appraisal tools. Available: https://joannabriggs.org/critical_appraisal_tools [Accessed 23 Oct 2019]. 